\title{
ALGORITM FOR DIAGNOSTICS OF APPENDICITIS IN CHILDHOOD AND SIRS
}

\author{
Y. Dimcheva ${ }^{1^{*}}$, Kr. Kalinova ${ }^{2}$, K. Georgiev ${ }^{2}$ \\ ${ }^{1}$ Department of Surgery, University Hospital -Deva Maria, Burgas, Bulgaria \\ ${ }^{2}$ Department of Pediatric Surgery, Medical Faculty, Trakia University, Stara Zagora, Bulgaria
}

\begin{abstract}
The specific purpose of this study was to describe and characterize the systemic inflammatory response to appendicitis in childhood. The clinical symptoms of SIRS are present in a large proportion of patients . A study of high-risk patients showed that over a given period of time, 44-68\% of the patients met the criteria for this condition, while at the same time they had proven infection up to 50\%.The incidence of SIRS is even higher in the post-operative period and in trauma regardless of the presence or absence of infection. On the other hand, between $10 \%$ and $43 \%$ of patients with proven sepsis do not meet the SIRS criteria.The inclusion of a number of biological markers (C-reactive protein, procalcitonin, cytokines) aims to help differentiate SIRS with infectious and noninfectious etiology. Sixty six patients were studied, divided into four groups from onset of symptoms to diagnosis. The primary outcome measure was to determine the systemic inflammatory response to appendicitis according to the established groups of time intervals. The secondary outcome measure was the analysis of C-reactive protein for the same purpose. The variables of the systemic inflammatory response, according to diagnostic intervals, showed nonsignificant differences in white blood cell count. The temperature rose constantly after $48 \mathrm{~h}$, reaching its peak after $72 \mathrm{~h}(\mathrm{p}=0.001)$, and the respiratory rate rose after $72 \mathrm{~h}(\mathrm{p}<0.0001)$. After $73 \mathrm{~h}$, most patients had three or four systemic inflammatory response criteria $(\mathrm{p}<0.0001)$. C-reactive protein levels rose progressively, showing higher levels after $48 \mathrm{~h}(\mathrm{p}=0.005)$. The inflammatory response to appendicitis is progressive, being more marked along the timeline from onset of symptoms to diagnosis.

Key words: appendicitis, diagnostic SIRS, children, algoritm.
\end{abstract}

\section{INTRODUCTION}

Systemic Inflammatory Response Syndrome (SIRS) reflects an inflammatory response to injury and infection [1, 2, 3, 5]. Systemic Inflammatory Response Syndrome (SIRS) is a physiological disorder that is non-specific, but is believed to be present in sepsis. Although the presence of SIRS is described in appendicitis patients, its progressive response to the development of clinical symptoms is not sufficiently described and characterized. Prolonged systemic inflammation in patients with disabilities and infectious processes can lead to multiple organ dysfunction and failure $[4,9]$. As with all acute inflammatory conditions, the ALA of appendicitis patients will develop and become more pronounced. The specific purpose of the study is to describe the IAS from the onset of the symptoms to the diagnosis in appendicitis children undergoing

\footnotetext{
*Correspondence to: Yanka Dimceva Department of Pediatric Surgery, Medical Faculty, Trakia University, Stara Zagora, Bulgaria e-mail: yanka_dimcheva@abv.bg
}

urgent surgery and to develop an adequate exemplary algorithm for children.

\section{MATERIAL AND METHODS}

A prospective, descriptive, study of 66 operated children with appendicitis in the Clinic of General and Operative Surgery, UMHAT - Stara Zagora and HK-UMBAL Deva Maria-Bourgas for the last 3 years was carried out. The tested children $(100 \%)$ were selected according to the following criteria: patients aged over 10 years subject to urgent appendectomy confirmed by histology. Patients were divided into four groups according to the time interval calculated from the onset of the symptoms as indicated by the patient to diagnosis: group 1: 0 to 24 hours, group 2: 25 to 48 hours, group 3: 49 to 72 hours, and group 4 more than 73 hours. Demographics and clinical data, a time interval from the onset of symptoms to diagnosis, white blood cell count (CBC), C-reactive protein (CRP), and SIRS values have been recorded. The main baseline measure is to determine the expected systemic inflammatory response of 
appendicitis according to the established groups at time intervals using the SIRS criteria for this purpose. Secondary baseline is the analysis of CRP as another inflammatory parameter, which is usually included in the
DIMCHEVA Y., et al. diagnostic trial of patients with appendicitis suspected.

\section{RESULTS}

Table 1. Frequency of CSF and CRP levels at different intervals

\begin{tabular}{lcccc} 
Variable & Group 1 $(0-24 \mathrm{~h})$ & Group 2 $(25-48 \mathrm{~h})$ & Group 3 $(49-72 \mathrm{~h})$ & Group 4 $(>73 \mathrm{~h})$ \\
\hline Leuco $(\mathrm{mm} 3)$ & $16,020 \pm 3,784$ & $17,630 \pm 3,780$ & $18,037 \pm 5,759$ & \\
$19,372 \pm 6,635$ & & & \\
Temperature $\left({ }^{\circ} \mathrm{C}\right) 37.7 \pm 0.4$ & $37.8 \pm 0.5$ & $37.8 \pm 0.9$ & $38.4 \pm 0.6$ \\
Heart/ min & $91.3 \pm 14.1$ & $106.4 \pm 14.6$ & $110 \pm 22.5$ & $125.8 \pm 16$ \\
Brith $(\mathrm{min})$ & $16.4 \pm 1.4$ & $16.9 \pm 1.4$ & $18.3 \pm 1.3$ & $22.6 \pm 2.8$ \\
CRP $(\mathrm{mg} / \mathrm{dL})$ & $162.7 \pm 95$ & $210.5 \pm 142$ & $231 \pm 121$ & $257.4 \pm 109$
\end{tabular}

$\mathrm{p}>0.0001$

There is no characteristic peritoneal irritation in the ileocecal region in the appendicitis retrocervical appendicitis (Figure 1). The response to the examination is inconsistent and does not correlate with the pronounced intoxication syndrome. There is a positive percussion on the right and different intensity of the right urothelial tenderness, more readily available for ultrasound diagnosis. Single hydroelectric levels are detected in X-ray examination. High comprehensiveness is attributed to CT use.

In the case of small-dose appendicitis (Figure 2), there are forewarned dysuria complaints, positive urine protein and microscopic hematuria (mean 9-11 erythrocytes in the sediment). Ultrasound and CT are highly informative. In cases of sub hepatic located appendicitis (Figure 3), there is a strong pronounced upper dyspeptic syndrome, as well as gastroenteritis manifestations. Laboratory studies show the highest leukocytosis - an average of 24000. The echography in this place of the inflamed appendix shows free moving fluid sub hepatic and the X- research - the HAC.

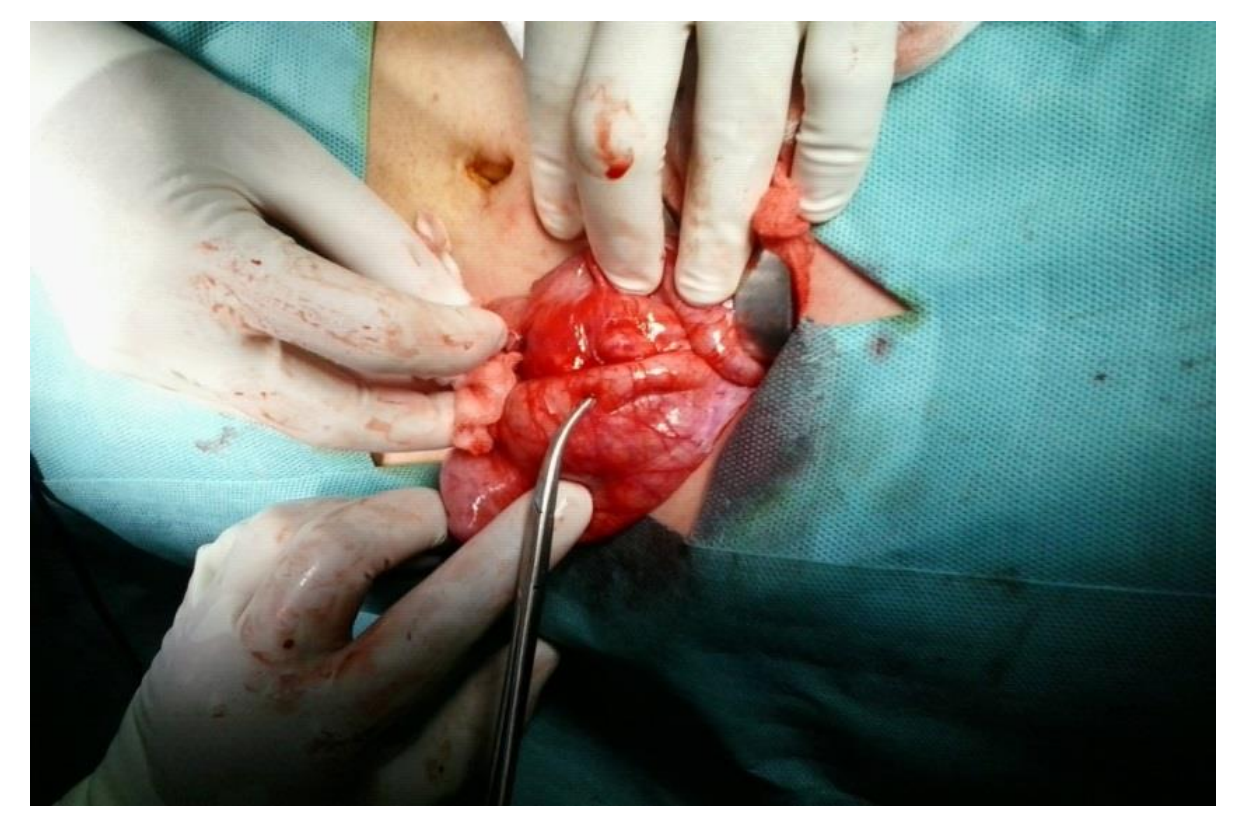

Figure 1. Retrocephalic catarally altered appendix

The clinical picture of retroperitoneal appendicitis (Figure 4) is governed by the general manifestations of fatigue, intoxication and dehydration, which are rapidly exacerbated. The localized symptoms are inconclusive, the ultrasound examines free movement of the liquid in the small pelvis, and $\mathrm{X}$-ray shows a number of XAC fine-grained origins. Leukocytosis is moderate. 


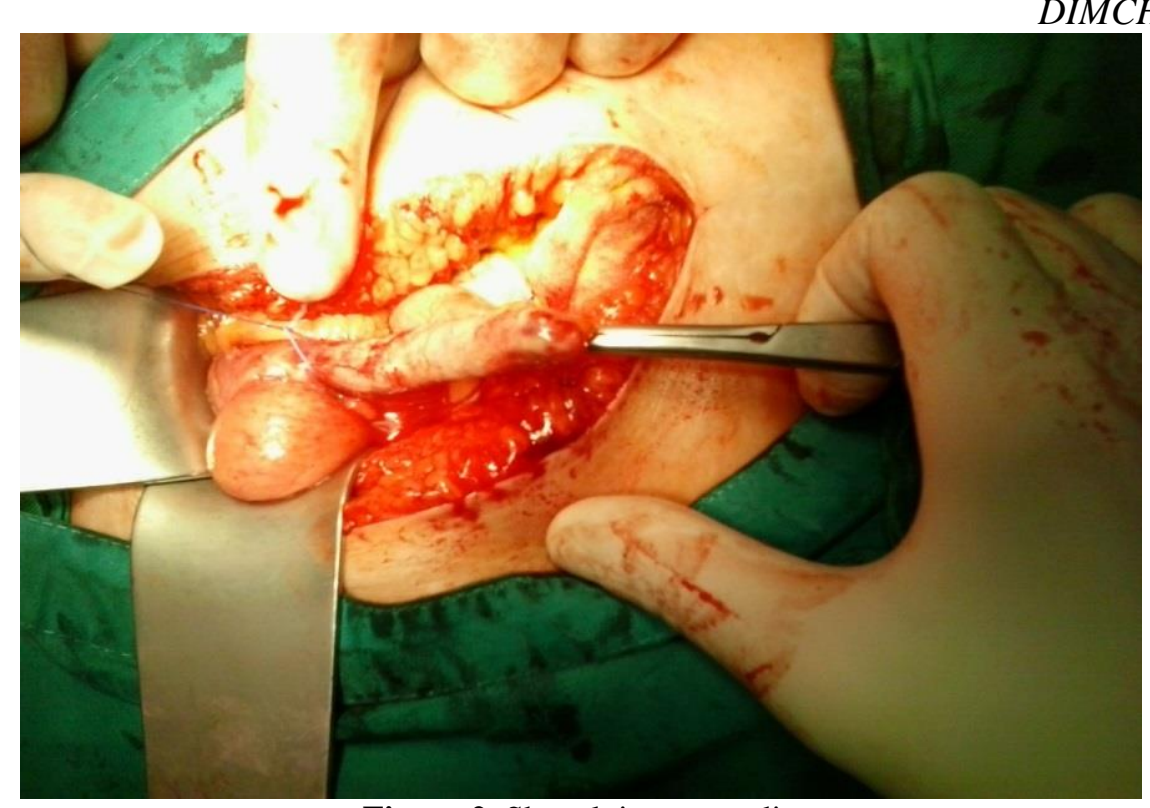

Figure 2. Short-lying appendix

Perforatives appendicitis incidence rates were $7 \%$ for group 1, 24\% for group 2, 53\% (p $<0.0001$ ) for group 3 and $85 \%(\mathrm{p}<0.0001)$ for group 4; it should be noted that the incidence of morbidity progresses over the time line, increasing significantly at each established diagnostic interval. The total perforation rate was $37 \%$. The SIRS perforation rate is $31 \%$ for one SIRS point, $20 \%$ for two SIRS points, $66 \%$ ( $\mathrm{p}<0.0001$ ) for three SIRS points and 80\% (p
$<0.0001)$ for four SIRS points; patients with SIRS 3 and 4 have the highest percentages of perforation. Correlations between perforation and PWC scores assessed by the Pearson test indicate that the perforation rate is correlated with the increased time interval and the higher SIRS score ( $\mathrm{p}<0.0001)$. Concerning the degree of complication only five complications (4\%); patients have a superficial surgical infection not requiring surgical interventions.

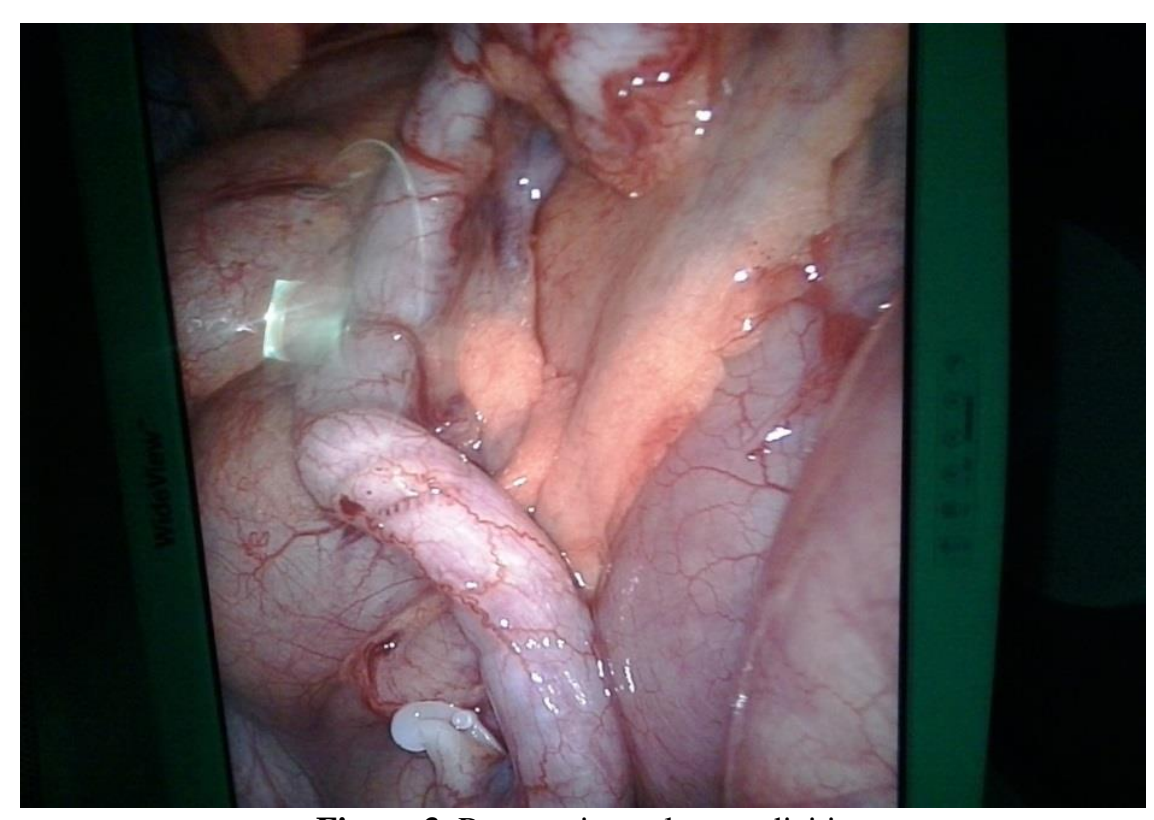

Figure 3. Retroperitoneal appendicitis

Inflammation of the blistering incidence of the cecum is the most common acute inflammatory disease in children. The higher incidence of complications with systemic inflammatory response (SIRS) childhood appendicitis is due to the following anatomical and physiological features:

- a smaller volume of the abdominal cavity and a closer proximity to the appendix with the other abdominal organs
- more mobile, and, in some cases, malaria - a lesser and less developed omenum that cannot limit and localize inflammation

- a senile immune system and a tendency to rapidly develop the inflammatory process and generalize it

- more often atypical, retro-cervical, subhepatic, small-dose, or retroperitoneal. 


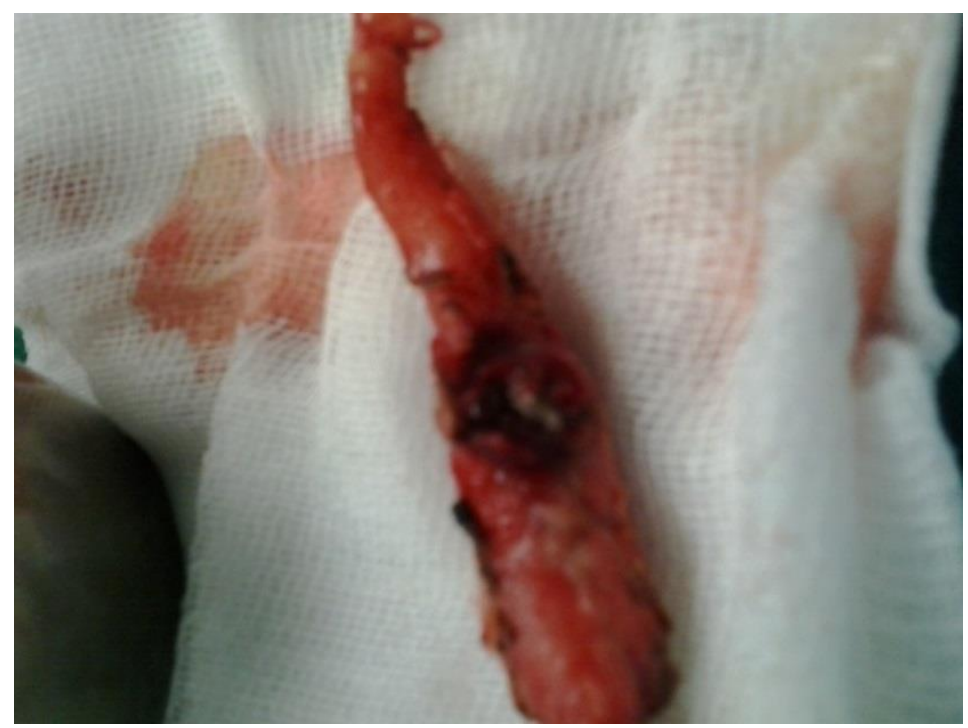

Figure 4. Retroperitoneally located appendicitis

For the presence of SIRS / SIRS / we speak when at least two or more of the following criteria exist (6-9):

1. fever-fever over 38 degrees or hypothermia below 36 degrees.

2. Changes in leukocyte-leukocytosis counts above 12,000 or Leucopenia below 4,000

3. Respiratory rate-tachypnea-above $20 \mu \mathrm{g} /$ min and RAC2 below $32 \mathrm{mg} \mathrm{Hg}$-this results in respiratory alkalosis, which is compensated for by metabolic acidosis

4. heart rate-tachycardia-over $90 \mathrm{~W} / \mathrm{min}$

5. Lactation - presence of more than $10 \%$ immature neutrophils in the peripheral blood PCK.

The clinical relevance of SIRS in patients with appendicitis has been investigated by comparing SIRS results with perforated appendicitis and post-operative complications.

The manifestation of WHO syndrome, as a reaction to an inflammatory process in the body, shapes the clinical picture of sepsis.

Difficulties in diagnosing complications of appendicitis in children arise due to the following features:

- Inability to assist patients in the group up to 3 years of age, restlessness, fear, weeping;

- the functional immaturity of the nervous system in young children, with all acute illnesses having a similar clinical picture gestation, vomiting, fever;

- the appendix's inflammation is violent and tends to spread the process;

- the markedly more atypical location of appendicular growth leads to less pronounced disease syndrome at the usual site and the appearance of symptoms resembling a number of other diseases of adjacent organs.
The importance of rapid diagnosis and treatment in patients with appendicitis is emphasized in order to avoid septic complications that increase with advanced pathology $[2,5,11,12]$. With an increase in the interval between onset of symptoms and surgery over 72 hours, the probability of a more severe pathology is higher than the interval below 12 hours $[2,3,5,6,11,12]$. The results of this study show elevated rates of CVD and diagnostic intervals associated with increased percentage of perforated appendicitis. The patient's delay in presenting to the emergency department after the onset of symptoms is associated with a worsening of the pathology as compared to internal delays [11]. Although these data support the importance of a progressive inflammatory response to appendicitis, proper inflammatory progression is not characterized [12]. This is important due to the recent assertions of conservative treatment of appendicitis [13-19], the high degree of conservative failed therapy and recurrent appendicitis, suggesting a problem with the design of these studies using unselected sequential samples with different diagnostic intervals, to take into account the fact that appendicitis is a developing disease, which deteriorates over time.

\section{Heart rhythm}

Increased heart rhythm is expected to be associated with the degree of fever; both responses mediated by the local and systemic production of serum inflammatory mediators such as phospholipase A2 and interleukins [2, 4]. The heart rate reaches higher values quickly after 24 hours of symptoms, staying high in all groups. After 73 hours most patients had high frequency tachycardia and this finding 
correlates with the elevated temperature and septic state of patients with longer diagnostic intervals.

\section{Breathing frequency}

Increased breathing rate after 72 hours means a severe septic state and metabolic acidosis. The frequency of breathing is the least common SIRS criteria met by the whole series; it had a significantly higher incidence in patients with a diagnosis interval greater than 73 hours. A frequency of $34 \%$ for this criterion was reported in a group of patients with mixed diagnostic intervals $[2,6,7]$, which is higher than the incidence found in this study where patients with higher diagnostic intervals are less frequent cases.

CRP is a non-specific inflammatory marker useful for maintaining the clinical diagnosis of appendicitis together with WBC and clinical parameters $[3,5,6,8]$. Higher values of CRP strongly correlate with appendicitis $[3,4,6$, 17]. For a longer diagnostic interval, CRP values were significantly higher $[3-6,12,20]$. The higher values of CRP have been shown to correlate with perforated appendicitis $[5,6$, 18]. Therefore, CRP is considered to be a better serological marker for advanced appendicitis than WBC [4-6, 19]. In this study, CRP values increased steadily with the diagnostic interval, reaching higher values after 48 hours, confirming previous observations indicating that CRP values were higher in advanced disease and a diagnostic interval higher than 48 hours. Given all these peculiarities, the following was outlined

\section{Sample algorithm for diagnosing SIRS complications in childhood}

I. Diagnostic Period-before Hospitalization:

-basal history, clinical examination:

-consultation of surgeon-palpation, auscultation, rectal tumors, temperature measurement and pediatrician consultation

If there is a doubt about the presence of a relevant pathology, consultations are needed: gynecologist for girls, gastroenterologist, and child cardiologist

-laboratory studies: CRC with leukogram, $\mathrm{CRP}$, urine; haemoculture

Regurgitation of the abdomen - radiographic radiography and radiography of the lung - at discretion;

-UZD / CT of abdominal organs;

II. Diagnostic period after hospitalization:

- the child is placed under active surveillance;

- Biochemical investigations are performed urea, creatinine, AST, ALT, bilirubin - direct and direct, ionogram, $\mathrm{ESR}, \mathrm{AF}$, total protein and albumin;

- control organ of the abdominal organs;

- Control imaging studies - at discretion

- if there is doubt in the diagnosis of more than 12 hours of hospitalization-diagnostic laparoscopy

\section{CONCLUSION}

The particularities of the child's organism, especially in younger age, determine the diagnostic difficulties and delay of the operative treatment and hence the higher incidence of complicated appendicitis in children. In childhood, the atypical location of the appendix is common, which is why for the appearance of an atypical clinical picture. The presence of an OSH is found in a significant number $(60.7 \%)$ of the infected children. Taking into account the specifics of the child's organism, especially in younger age, in the case of prominent symptoms of Systemic Inflammatory Response Syndrome, complicated appendicitis should be considered and the algorithm used to diagnose or reject this disease.

\section{REFERENCES}

1. Witaliewna Olga Associated forms of appendicular peritonitis in childhood.Diss. 2006r.

2. Evstatieva, Irina Iv. Laparoscopy in diagnostics and treatment of organ malignancies, simulating the acute appendicitis in the child. Autoreff. Higher Medical Academy, 2008.

3. Drozdow.G Ultrasound diagnostic of appendicitis and its complications. Diss.Moscow. 1996.

4. Ahmetchin RL., A.A.Boldjir, PA Bolzjir and others.Emergency surgery and biotechnology Clinical Russian FederationDonetzc, 2013.

5. Karapetian E.Clinical immunological characteristic of appendicular peritonitis in childhood. Diss.Moscow 2005.

6. Stephenson JA, Gravante G, Butler NA, Sorge R, Sayers RD, Bown MJ. The systemic inflammatory response (SIRS) number and type of positive criteria predict interventions and outcomes in acute surgical admissions. World $J$ Surg. 34:2757-2764, 2010.

7. Kirsi-Maija Kaukonen, Michael Bailey, David Pilch Jamie Cooper, and Rinaldo Bellomo Systemic Inflammatory Response Syndrome Criteria in Defining Severe Sepsis.N Engl J Med; 372:1629-1638, 2015.

8. Raines A1, Garwe T, Wicks R, Palmer M, Wood F, Adeseye A, Tuggle D. 
Pediatric appendicitis: the prevalence of systemic inflammatory response syndrome upon presentation and its association with clinical outcomes. $J$ Pediatr Surg. Dec;48(12):2442-5, 2013.

9. M. Sigfrido Rangel-Frausto, Didier Pittet, Michele Costigan et al The Natural History of the Systemic Inflammatory Response Syndrome (SIRS) A Prospective Study JAMA.; 273(2):117-123, 1995.

10.Chawla BK, Teitelbaum DH: Profound systemic inflammatory response syndrome following non-emergent intestinal surgery in children. J Pediatr Surg. 48(9):1936-40, 2013.

11.Miyano, G., Okazaki, T., Kato, Y. et al.Open versus laparoscopic treatment for pan-peritonitis secondary to perforated appendicitis in children: a prospective analysis. $J$ Laparoendosc Adv S. 20:655657, 2010.

12.Becker, T., Kharbanda, A., Bachur, R. Atypical clinical features of pediatric appendicitis. Acad Emerg Med. 14:124129, 2007.

13.AnderssonR.E. Meta-analysis of the clinical and laboratory diagnosis of acute appendicitis. Br J Surg.;1:28-37, 2004.

14.Ceydeli A, Lavotshkin S, Yu J, et al. When should we order a CT scan and when should we rely on theresults to diagnose an acuteappendicitis? CurrSurg.63:464-468, 2006.
DIMCHEVA Y., et al.

15.Bendeck SE, Nino-Murcia M, Berry GJ, et al. Imagingfor suspected appendicitis: negative appendectomy and perforationrates.Radiology.225:131-136. 2002.

16.Musunuru S, Chen H, Rikkers LF, et al. Computed tomography in the diagnosis of acute appendicitis: definitive ordetrimental? $J \quad$ Gastrointest Surg.;11:1417-1421, 2007.

17.Terasawa $\mathrm{T}$, Blackmore $\mathrm{CC}$, Bent $\mathrm{S}$, et al. Systematic review: computed tomography and ultrasonography to detect acute appendicitis in adults and adolescents. AnnInternMed.141:537-546, 2004.

18. Society of American Gastrointestinal and Endoscopic Surgeons (SAGES). Guidelines for diagnosis, treatment, and use of laparoscopy for surgical problems during pregnancy. Los Angeles (CA): Society of American Gastrointestinal and Endoscopic Surgeons (SAGES); 31 p. 2011.

19.Solomkin J.S., Mazuski J.E., Bradley J.S. et al. Diagnosis and management of complicated intra-abdominal infection in adults and children: guidelines by the Surgical Infection Society and the Infectious Diseases Society of America. Clin Infect Dis Jan 15;50(2):133 -64, 2010. 